\title{
Mucosal eosinophilia and recurrence of nasal polyps - new classification of chronic rhinosinusitis*
}

\author{
Tsuguhisa Nakayama, Mamoru Yoshikawa, Daiya Asaka, Tetsushi Okushi, \\ Yoshinori Matsuwaki, Nobuyoshi Otori, Takanori Hama, Hiroshi Moriyama \\ Department of Otorhinolaryngology, Jikei University School of Medicine, Tokyo, Japan
}

SUMMARY Background: Eosinophils and nasal polyps are believed to affect the surgical outcome of chronic rhinosinusitis (CRS). CRS is classified based on the presence of nasal polyps in western countries. The majority of patients with CRS with nasal polyps (CRS with NP) are characterized by predominantly eosinophilic inflammation. However, Asian patients with CRS with NP show characteristics indicative of neutrophilic inflammation. Therefore, are eosinophils or nasal polyps more important for the classification of CRS?

Methods: A prospective cohort study conducted from April 2007 to March 2008 classified patients with CRS based on the presence of nasal polyps and mucosal eosinophilia. The recurrence rate of nasal polyps was compared between the groups. Recurrence rate was analysed as a time-dependent variable by the Kaplan-Meier method.

Results: Eosinophilic inflammation was found in $59.6 \%$ of patients with CRS with NP. Patients with mucosal eosinophilia had higher polyp recurrence rate than patients without mucosal eosinophilia, whereas patients with nasal polyps did not have higher polyp recurrence rate than patients without nasal polyps.

Conclusions: Presence of mucosal eosinophilia is a more important factor than nasal polyps for classifying CRS in terms of the surgical outcome.

Key words: chronic rhinosinusitis, eosinophils, nasal polyps, endoscopic sinus surgery, recurrence

\section{INTRODUCTION}

Chronic rhinosinusitis (CRS) is one of the most common chronic diseases, but little is understood about its pathogenesis. CRS is usually classified based on the presence or absence of nasal polyps ${ }^{(1,2)}$. The presence of nasal polyps indicates refractory disease with a tendency to recur, often requiring long-term medical therapy despite successful surgical intervention ${ }^{(3-5)}$. Mucosal eosinophilia is also widely reported as a marker of inflammation in patients with CRS ${ }^{(6)}$. The presence of mucosal eosinophilia is frequently associated with more severe disease and recurrence of nasal polyps after surgery ${ }^{(7)}$. However, nasal polyps do not show the same eosinophilic inflammatory pattern in different parts of the world (8). Eosinophilic inflammation is found in about $80 \%$ of cases of CRS with nasal polyps (CRS with NP) in western countries (9). In contrast, neutrophilic inflammation is common in cases of CRS with NP in Asia, such as south Chinese and Korean patients ${ }^{(10,11)}$. Such findings raise the question of which characteristic is more important for the classification of CRS, the presence of eosinophils or nasal polyps?
The present study investigated the effect of mucosal eosinophilia and nasal polyps on the recurrence rate of nasal polyps and the importance of these characteristics for the classification of CRS.

\section{METHODS}

Subjects

This prospective study enrolled 223 patients with CRS from April 2007 to March 2008. The diagnosis of sinus disease was based on patient history, clinical examination, nasal endoscopy, and computed tomography (CT) of the sinuses according to the guidelines of the European Position Paper on Rhinosinusitis and Nasal Polyps ${ }^{(1)}$. This study excluded patients treated with oral steroid or antimicrobial agents within 4 weeks before surgery, and patients with unilateral disease, fungal disease, antrochoanal polyps, and cyst of the paranasal sinuses. Preoperative demographic and medical history was obtained from the patient, including age, sex, history of prior sinus surgery, smoking habit, asthma, and allergic rhinitis. Allergic rhinitis was confirmed by intradermal skin testing, and 
serum total immunoglobulin E (IgE) and specific IgE for common allergen were measured by fluoroenzyme immunoassay. CT findings were graded according to the Lund-MacKay method ${ }^{(12)}$. All patients followed up for at least 6 months were included in the analysis. Polyps were graded using the 0 - 3 scoring system recommended by the guidelines: score 0 , absence of polyps; score 1, polyps in middle meatus only; score 2, polyps beyond the middle meatus but not blocking the nose completely; score 3 , polyps completely obstructing the nose ${ }^{(1)}$. Recurrence of CRS was defined as the presence of nasal polyps detected by nasal endoscopy. The study was approved by the ethics committee of Jikei University School of Medicine.

\section{Symptom scores}

All participants were assessed before surgery regarding 5 symptoms; nasal obstruction, anterior nasal drainage, posterior nasal drainage, facial pain, and decreased sense of smell. The severity of each symptom was evaluated according to the 7-point (score 0 - 6) Likert scale. Total symptom score was calculated as the total of the 5 symptom scores.

\section{CRS subgroups}

Patients with CRS were classified on the basis of the presence of nasal polyps and histological detection of mucosal eosinophilia as defined by the eosinophil cut point into the following groups: eosinophilic CRS with nasal polyps (ECRS with NP), eosinophilic CRS without nasal polyps (ECRS without NP), non-eosinophilic CRS with nasal polyps (NECRS with NP), and non-eosinophilic CRS without nasal polyps (NECRS without NP). The recurrence of nasal polyps in these groups was investigated after endoscopic sinus surgery.

\section{Histological analysis}

Mucosal tissues of patients with CRS were removed from the nasal polyps or mucosa of the ethmoid cavity at the time of surgery. The tissue was fixed immediately in $10 \%$ formalin, embedded in paraffin, and cut into thin sections. The sections were stained with hematoxylin-eosin stain. The number of eosinophils in the mucosa was counted under high-power field $(\mathrm{HPF}, \times 400)$ in which eosinophils were the densest cellular infiltrate beneath the epithelial surface. Histological examinations were performed by three physicians unaware of the clinical data, and the mean number of eosinophils was calculated.

\section{Statistics}

The eosinophil cut off point was determined from the relationship between the eosinophil count and polyp recurrence using the univariate Cox proportional hazards model and the area under the receiver operating characteristic curve (AUC). The optimal cut off point was defined with the minimum corresponding $\mathrm{p}$-value and the maximum AUC. Patients with eosinophil count above the cut off point were considered to have mucosal eosinophilia. The analysis of variance test was used to evaluate differences in patient characteristics between the subgroups. Disease-free survival curves were drawn using the
Kaplan-Meier method and compared using the log-rank test. Disease-free survival was defined as the time that the patient remained without nasal polyps after endoscopic sinus surgery. Cox proportional hazard models were fitted for subgroups and parameters, and hazard ratio (HR) and 95\% confidence intervals (CI) were calculated. All statistical analyses were performed using STATA 9.1 (STATA Corp, College Station, TX, USA). A value of $\mathrm{p}<0.05$ was considered statistically significant.

\section{RESULTS}

Patient characteristics

A total of 175 of the 223 enrolled patients (78.5\%) had adequate data for analyses. The 129 male and 46 female patients were aged 19 to 77 years (mean 48.1 years). The mean followup period was 17.5 months. Twenty-one $(12.0 \%)$ of these patients had asthma, $90(51.4 \%)$ had allergic rhinitis, and 50 $(28.6 \%)$ were current smokers. Forty patients $(22.9 \%)$ suffered polyp recurrence after surgery during the follow-up period.

\section{Eosinophil cut off point}

The associations between eosinophil count and polyp recurrence are shown in Figure 1. Patients with $\geq 60$ and $\geq 70$ eosinophils/HPF showed the minimum $\mathrm{p}$ values $(\mathrm{p}=0.001)$ and patients with $\geq 70$ eosinophils/HPF showed the highest AUC (0.673). Therefore, mucosal eosinophilia was defined as $\geq 70$ eosinophils/HPF.

\section{CRS subgroups}

Clinical characteristics of each classification are summarized in Table 1. More than half (59.6\%) of the patients with CRS with NP had mucosal eosinophilia. The numbers of patients with allergic rhinitis $(p=0.019)$, asthma $(p=0.001)$, current smoker $(p=0.032)$, and total symptom score $(p=0.005)$ were significantly different between the 4 groups. The number of patients with polyp recurrence was also significantly different between these groups $(\mathrm{p}=0.001)$.

\section{Prognostic factors}

Patients with mucosal eosinophilia (70/HPF and over) had a poor prognosis (HR: 3.47; 95\% CI: 1.65-7.29; $\mathrm{p}=0.001$ ). Asthma $(p=0.000)$, polyp score $(p=0.001)$, CT score $(p=0.001)$, and allergic rhinitis $(p=0.026)$ were also predictors of recurrence by univariate analysis using the Cox proportional model (Table 2). The real eosinophil numbers were not correlated with recurrence (HR: 1.001; 95\% CI: 0.99-1.00; $\mathrm{p}=0.164)$.

\section{Recurrence rate}

Patients with mucosal eosinophilia had a significantly higher polyp recurrence rate by log-rank test $(p=0.0005)$ (Figure 2A). However, patients with nasal polyps did not have a significantly higher polyp recurrence rate than patients without nasal polyps $(\mathrm{p}=0.0535)$ (Figure $2 \mathrm{~B})$. Regardless of the presence or absence of nasal polyps, patients with mucosal eosi- 


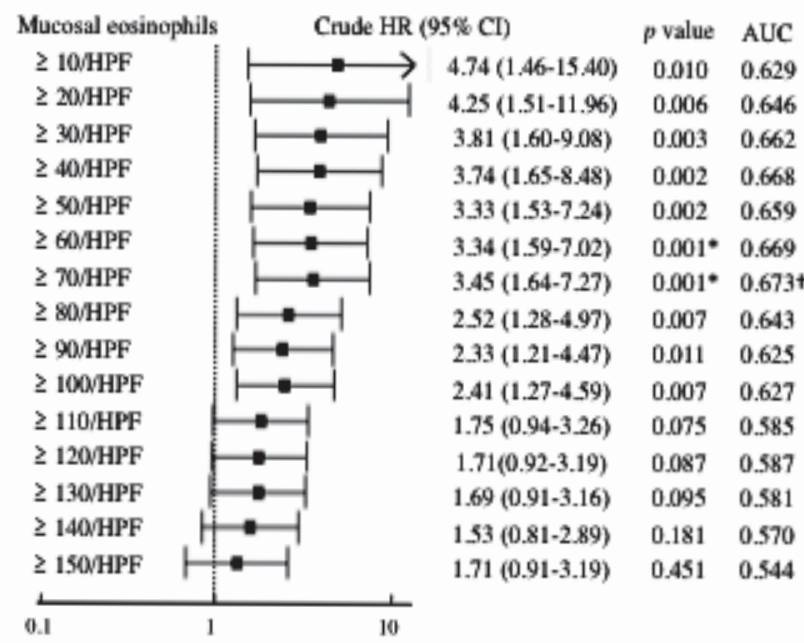

Figure 1. Association of mucosal eosinophils with polyp recurrence. $\mathrm{HR}=$ hazard ratio, $\mathrm{CI}=$ confidence interval. ${ }^{*}$ Minimum $\mathrm{p}$ value, †highest AUC.

nophilia had a significantly higher recurrence rate $(\mathrm{p}=0.0067)$ among the 4 groups (Figure 2C). Investigation of disease-free survival in the 4 groups showed that patients with NECRS with NP (HR, 0.27; 95\% CI, 0.10-0.72; p = 0.008) and NECRS without NP (HR, 0.24; 95\% CI, 0.10-0.72; $p=0.007$ ) had better prognosis than patients with ECRS with NP. Patients with ECRS without NP (HR, 0.55; 95\% CI, 0.21-1.44; $\mathrm{p}=0.225$ ) did not have better survival than patients with ECRS with NP (Table 3). These findings indicate that the presence of mucosal eosinophilia is more important than nasal polyps in the recurrence of CRS.

\section{DISCUSSION}

The present study found that more than half $(59.6 \%)$ of Japanese patients with CRS with NP had mucosal eosinophilia. Histologically, CRS with NP is believed to be characterized by predominantly eosinophilic inflammation, whereas CRS without NP is characterized by neutrophilic inflammation with a lesser contribution of eosinophils ${ }^{(1,2)}$. Eosinophilic inflammation has been reported in $80 \%$ of patients with CRS with NP ${ }^{(9)}$, suggesting that CRS with NP is equivalent to CRS with mucosal eosinophilia. However, mucosal eosinophilia was present in $46.4 \%$ of patients with CRS with NP versus $12 \%$ of patients with CRS without NP in south China ${ }^{(10)}$. Moreover, eosinophilic inflammation was found only in 33.3\% of 30 patients in Korea ${ }^{(11)}$. Therefore, NPs are not associated with the same eosinophilic inflammatory patterns in different parts of the world ${ }^{(8)}$. This study showed that the prevalence of mucosal eosinophilia in Japanese patients with CRS with NP was intermediate between the prevalences reported in western countries and other eastern Asian countries.

The presence of mucosal eosinophilia is frequently associated with more severe disease and recurrence of nasal polyps after
A

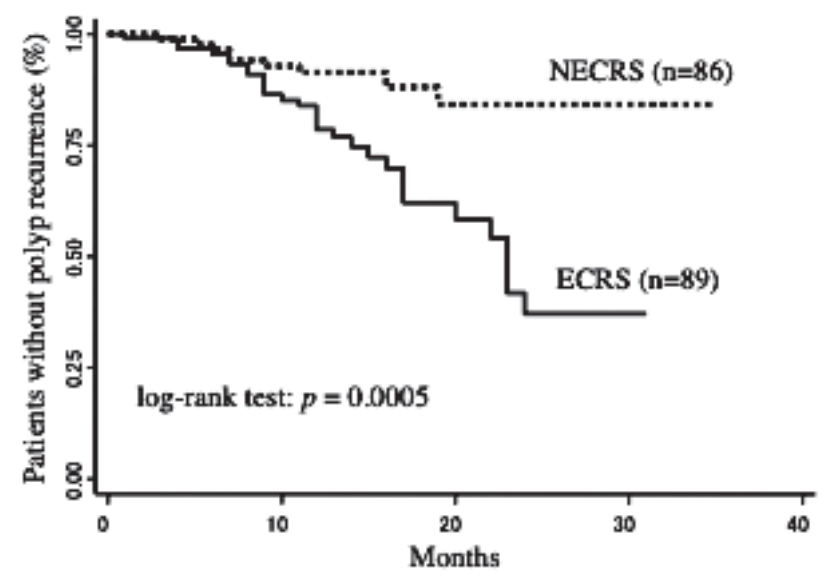

B

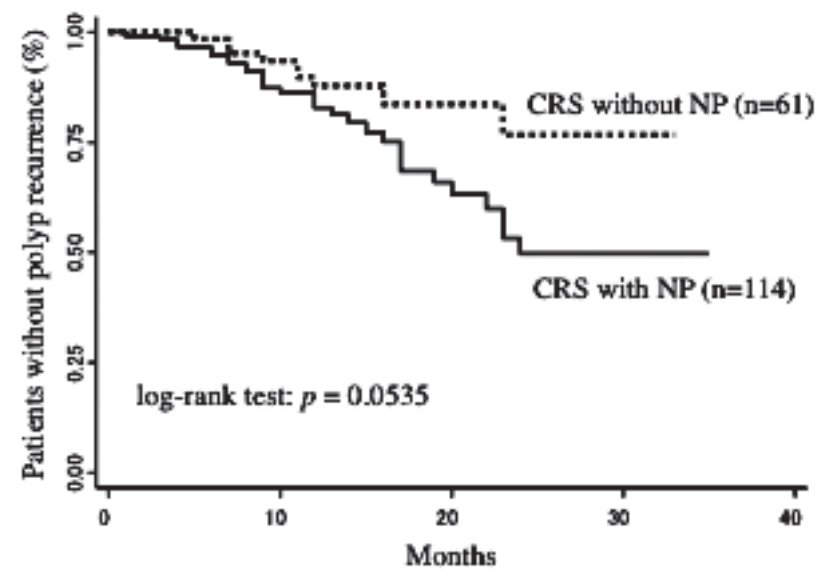

C

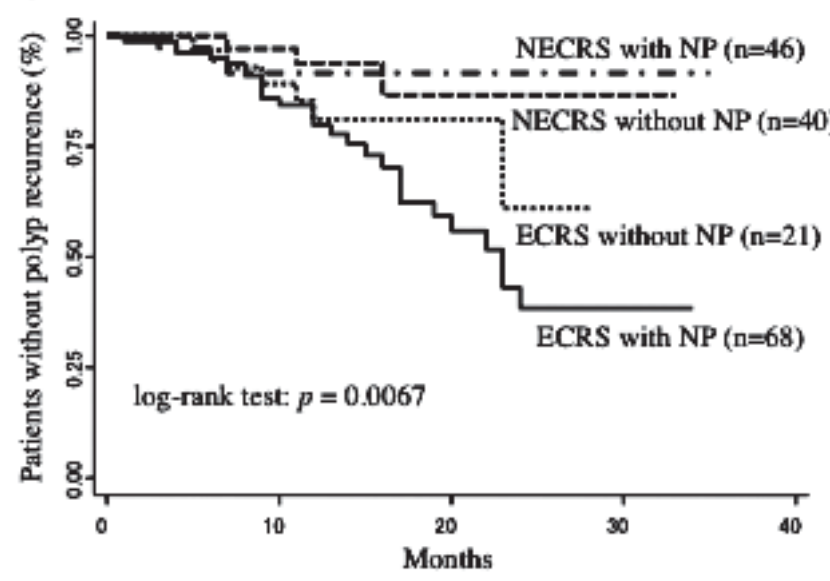

Figure 2. Kaplan-Meier curves of disease-free survival rates in patients with CRS. (A) Survival of patients with mucosal eosinophilia (ECRS) (solid line) exceeded survival of patients without mucosal eosinophilia (NECRS) (dashed line). (B) Survival of patients with nasal polyps (CRS with NP) (solid line) exceeded survival of patients without nasal polyps (CRS without NP) (dashed line), not significant. (C) Survival of patients with mucosal eosinophilia (ECRS with NP and ECRS without NP) exceeded survival of patients without mucosal eosinophilia.

surgery ${ }^{(7)}$. Several studies have investigated the relationship between the number of mucosal eosinophils and surgical 
Table 1. Patient characteristics. Level of significance (p) obtained by analysis of variance.

\begin{tabular}{|c|c|c|c|c|c|}
\hline & $\begin{array}{l}\text { ECRS with NP } \\
\quad(\mathrm{n}=68)\end{array}$ & $\begin{array}{c}\text { ECRS without } \\
\text { NP } \\
(\mathrm{n}=21)\end{array}$ & $\begin{array}{c}\text { NECRS with NP } \\
\quad(\mathrm{n}=46)\end{array}$ & $\begin{array}{c}\text { NECRS without } \\
\text { NP } \\
(\mathrm{n}=40)\end{array}$ & $\mathrm{p}$ value \\
\hline Sex, female/male & $17 / 51$ & $6 / 15$ & $11 / 35$ & $12 / 28$ & 0.912 \\
\hline Age, mean $\pm \mathrm{SE}(\mathrm{y})$ & $48.2 \pm 13.3$ & $42.6 \pm 12.4$ & $51.1 \pm 16.8$ & $47.5 \pm 14.3$ & 0.172 \\
\hline Previous surgery & $14 / 68$ & $2 / 21$ & $10 / 46$ & $8 / 40$ & 0.679 \\
\hline Allergic rhinitis & $43 / 68$ & $13 / 21$ & $17 / 46$ & $17 / 40$ & 0.019 \\
\hline Asthma & $16 / 68$ & $3 / 21$ & $2 / 46$ & $0 / 40$ & 0.001 \\
\hline $\operatorname{IgE}(\mathrm{IU} / \mathrm{ml})$ & $277.6 \pm 364.6$ & $179.5 \pm 315.4$ & $178.9 \pm 358.8$ & $132.0 \pm 149.1$ & 0.131 \\
\hline CT score & $14.09 \pm 5.97$ & $9.00 \pm 3.38$ & $13.09 \pm 5.49$ & $7.65 \pm 3.48$ & 0.000 \\
\hline Polyp score & $4.00 \pm 1.58$ & $0.62 \pm 0.67$ & $3.59 \pm 1.47$ & $0.78 \pm 0.89$ & 0.000 \\
\hline Current smoker & $20 / 68$ & $1 / 21$ & $13 / 46$ & $16 / 40$ & 0.032 \\
\hline TSS & $15.22 \pm 5.12$ & $13.81 \pm 7.06$ & $14.57 \pm 7.11$ & $10.85 \pm 6.08$ & 0.005 \\
\hline Follow up (m) & $18.07 \pm 8.01$ & $18.62 \pm 7.71$ & $16.72 \pm 8.61$ & $16.80 \pm 7.23$ & 0.679 \\
\hline Polyp recurrence & $26 / 68$ & $5 / 21$ & $5 / 46$ & $4 / 40$ & 0.001 \\
\hline
\end{tabular}

TSS $=$ total symptom score.

Table 2. Cox proportional hazard models.

\begin{tabular}{lccc}
\hline Variable & Crude HR & $95 \%$ CI & $\mathrm{p} \mathrm{value}$ \\
\hline$\geq 70$ eosinophils/HPF & 3.47 & $1.65-7.29$ & $0.001^{*}$ \\
Polyp score & 1.30 & $1.12-1.52$ & $0.001^{*}$ \\
Asthma & 3.26 & $1.69-6.26$ & $0.000^{*}$ \\
Allergic rhinitis & 2.15 & $1.09-4.24$ & $0.026^{*}$ \\
CT score & 1.09 & $1.03-1.15$ & $0.001^{*}$ \\
Prior sinus surgery & 1.09 & $0.50-2.38$ & 0.819 \\
Current smoker & 0.53 & $0.23-1.20$ & 0.127 \\
TSS & 1.04 & $0.99-1.09$ & 0.149 \\
Age & 0.99 & $0.48-1.90$ & 0.915 \\
Male & 0.96 & $0.43-2.45$ & 0.951 \\
\hline
\end{tabular}

*p value is significant. $\mathrm{HR}=$ hazard ratio, $\mathrm{CI}=$ confidence interval, $\mathrm{TSS}=$ total symptom score .

Table 3. Cox proportional hazard model.

\begin{tabular}{lccc}
\hline Variable & Crude HR & $95 \%$ CI & p value \\
\hline ECRS with NP & Reference & - & 1.000 \\
ECRS without NP & 0.553 & $0.212-1.440$ & 0.225 \\
NECRS with NP & 0.274 & $0.104-0.715$ & $0.008^{*}$ \\
NECRS without NP & 0.235 & $0.082-0.674$ & $0.007^{*}$ \\
\hline
\end{tabular}

${ }^{*} \mathrm{p}$ value is significant. $\mathrm{HR}=$ hazard ratio, $\mathrm{CI}=$ confidence interval, $\mathrm{TSS}=$ total symptom score.

outcomes, but few studies have considered the level of tissue eosinophil density required to define mucosal eosinophilia. Mucosal eosinophilia was defined as $>10$ eosinophils/HPF and patients with eosinophilia showed significantly less improvement in quality-of-life outcomes ${ }^{(13)}$. Mucosal eosinophilia was also defined as more than 5 eosinophils/HPF and patients with mucosal eosinophilia had higher postoperative endoscopy scores ${ }^{(14)}$. The present study found that levels of mucosal eosinophil infiltrate of $\geq 70 / \mathrm{HPF}$ had the greatest impact on surgical outcome. Therefore, we defined mucosal eosinophilia as $\geq 70$ eosinophils/HPF. However, this cut off point for eosinophil concentration does not specify the patient popula- tion with CRS. Patients with asthma are often treated with steroids before sinus surgery, and such medical intervention affects mucosal inflammation, including the degree of mucosal eosinophilia. Therefore, we excluded patients treated with oral steroids. Consequently, the real cut off point may be higher than 70 eosinophils/HPF.

In this study, patients with CRS with NP tended to show higher polyp recurrence than patients with CRS without NP, but not reaching statistical significance. In contrast, patients with CRS with mucosal eosinophilia showed significantly higher polyp recurrence. Therefore, we considered that eosinophils 
are central in the pathogenesis of CRS. The pathophysiology of CRS with NP shows many differences from that of CRS without NP in western countries ${ }^{(1,2)}$. Mucosal eosinophilia is a characteristic of nasal polyps, but not of CRS without NP. However, we found that mucosal eosinophilia is also a key prognostic factor of CRS without NP. Interestingly, although patients with ECRS without NP did not have nasal polyps at the time of surgery, they showeda high polyp recurrence rate in the postoperative period. These findings suggest that eosinophilic CRS with NP and eosinophilic CRS without NP might be interpreted as different degrees of inflammation, and moreover may actually be the same disease entity. On the other hand, patients with non-eosinophilic with NP did not have a high polyp recurrence rate. In Chinese, CRS without NP and non-eosinophilic CRS with NP share a number of similarities in granulocyte activation and $\mathrm{Th}$ cell responses ${ }^{(10)}$, indicating that non-eosinophilic CRS with NP and non-eosinophilic CRS without NP may be the same disease entity.

CRS with NP is equivalent to CRS with mucosal eosinophilia in western countries. Therefore, CRS with NP has been considered to have a high polyp recurrence. In fact, the initial stage of sinonasal polyposis did not correlate with recurrence after surgery or long-term outcomes in the European population ${ }^{(15)}$. Distinct mechanisms have not been considered to underlie the pathogenesis of NPs in these regions.

The present study indicates that mucosal eosinophilia is a more important prognostic factor than the presence of nasal polyps in terms of the surgical outcome. Consequently, we conclude that mucosal eosinophilia is a more important factor for classifying CRS than nasal polyps.

\section{AUTHOR CONTRIBUTIONS}

TN: data collection, analysis, writer; MY: study design, data collection; DA: study design, data collection; TO: study design, data collection; YM: study design, data collection; NO: study design, data collection; TH: statistical analysis; HM: conduct

\section{CONFLICT OF INTEREST STATEMENT}

None of the authors have any conflict of interest, financial or otherwise.

\section{REFERENCES}

1. Fokkens W, Lund V, Mullol J. European Position Paper on Rhinosinusitis and Nasal Polyps Group. EP3OS 2007: European position paper on rhinosinusitis and nasal polyps 2007. A summary for otorhinolaryngologists. Rhinology. 2007; 45: 97-101.
2. Meltzer EO, Hamilos DL, Hadley JA, et al. American Academy of Allergy, Asthma and Immunology (AAAAI); American Academy of Otolaryngic Allergy (AAOA); American Academy of Otolaryngology--Head and Neck Surgery (AAO-HNS); American College of Allergy, Asthma and Immunology (ACAAI); American Rhinologic Society (ARS). Rhinosinusitis: establishing definitions for clinical research and patient care. J Allergy Clin Immunol. 2004; 114(6 Suppl): 155-212.

3. Deal RT, Kountakis SE. Significance of nasal polyps in chronic rhinosinusitis: symptoms and surgical outcomes. Laryngoscope. 2004; 114: 1932-1935.

4. Vento SI, Ertama LO, Hytönen ML, Wolff $\mathrm{CH}$, Malmberg $\mathrm{CH}$. Nasal polyposis: clinical course during 20 years. Ann Allergy Asthma Immunol. 2000; 85: 209-214.

5. Norès JM, Avan P, Bonfils P. Medical management of nasal polyposis: a study in a series of 152 consecutive patients. Rhinology. 2003; 41: 97-102.

6. Bhattacharyya N, Vyas DK, Fechner FP, Gliklich RE, Metson R. Tissue eosinophilia in chronic sinusitis: quantification techniques. Arch Otolaryngol Head Neck Surg. 2001; 127: 1102-1105.

7. Ferguson BJ. Categorization of eosinophilic chronic rhinosinusitis. Curr Opin Otolaryngol Head Neck Surg. 2004; 12: 237-242.

8. Zhang N, Holtappels G, Claeys C, et al. Pattern of inflammation and impact of Staphylococcus aureus enterotoxins in nasal polyps from southern China. Am J Rhinol. 2006; 20: 445-450.

9. Stoop AE, van der Heijden HA, Biewenga J, van der Baan S. Eosinophils in nasal polyps and nasal mucosa: an immunohistochemical study. J Allergy Clin Immunol. 1993; 91: 616-622.

10. Cao PP, Li HB, Wang BF, et al. Distinct immunopathologic characteristics of various types of chronic rhinosinusitis in adult Chinese. J Allergy Clin Immunol. 2009; 124: 478-484, 484.e1-2.

11. Kim JW, Hong SL, Kim YK, et al Histological and immunological features of non-eosinophilic nasal polyps. Otolaryngol Head Neck Surg. 2007; 137: 925-930.

12. Lund VJ, Mackay IS. Staging in rhinosinusitus. Rhinology. 1993; 31: 183-184.

13. Soler ZM, Sauer D, Mace J, Smith TL. Impact of mucosal eosinophilia and nasal polyposis on quality-of-life outcomes after sinus surgery. Otolaryngol Head Neck Surg. 2010; 142: 64-71.

14. Kountakis SE, Arango P, Bradley D, Wade ZK, Borish L. Molecular and cellular staging for the severity of chronic rhinosinusitis. Laryngoscope. 2004; 114: 1895-1905.

15. Garrel R, Gardiner Q, Khudjadze M, et al. Endoscopic surgical treatment of sinonasal polyposis-medium term outcomes (mean follow-up of 5 years). Rhinology. 2003; 41: 91-96.

Tsuguhisa Nakayama

Department of Otorhinolaryngology

Jikei University School of Medicine

3-25-8 Nishi-shinbashi, Minato-ku

Tokyo, 105-8461

Japan

Tel: +81-3-3433-1111

Fax: +81-3-3578-9208

E-mail: nakayama-t@jikei.ac.jp 